\title{
The Prevalence of Botnets in Taiwan and China
}

\author{
Dr Yao-chung Chang \\ Regulatory Institutions Network \\ The Australian National University \\ Canberra, Australia \\ Lennon.Chang@anu.edu.au
}

\author{
Dr Gregor Urbas \\ ANU College of Law \\ The Australian National University \\ Canberra, Australia \\ Gregor.Urbas@anu.edu.au
}

\begin{abstract}
This paper discusses the prevalence of bot-infected computers in Taiwan and China and why bot-infected computers are so prevalent in Taiwan and China. Reports have shown that Taiwan and China are the top two countries in the Asia-Pacific and Japan (APJ) region where bot-infected computers are located. However, there is still little empirical study on the reasons why China and Taiwan rank so high in terms of bot-infected computers. Based on interview data collected in 2008 and 2009 in the Greater China area, this paper analyses the prevalence of bot-infected computers in Taiwan and China from five perspectives: technical perspective, user perspective, political perspective, cultural perspective and business perspective.
\end{abstract}

\section{Keywords - botnet, bot-infected computers, Taiwan, China}

\section{INTRODUCTION}

A botnet is a group of networked computers with secretly installed programs that allow people to control those computers remotely. A computer infected with a malicious program or malware that allows it to be remotely controlled is bot-infected. A home or office computer may be bot-infected and actively being used as part of a botnet without the computer owner's knowledge. Using large numbers of net-worked infected-computers, botnet operators can launch highly damaging attacks, including such serious crimes as Distributed Denial of Service (DDoS) attacks. Some large botnets with hundreds of thousands of computers controlled have been found, and these can be employed for purposes of cyber-terrorism and cyber-war.

Reports have shown that, compared to other countries in the Asian and Pacific region, Taiwan and China are the top two countries where most bot-infected computers are located. Despite the consequences that botnets have brought to both countries, there are still little systematic analysis on the prevalence of bot-infected computers in Taiwan and China.

Supported by interview data collected in Taiwan and China in 2008 and 2009 by the first author, this paper will explain why Taiwan and China both rank highly on the worldwide list of bot-infected computers.

\section{PREVAlence OF Botnets In TAIWAN AND ChINA}

China and Taiwan are the countries which have the most bot-infected computers in the APJ region. China led in terms of bot-infected computers in the APJ region in 2007, followed by Taiwan (Table 1). In the second half of 2007, there were $43 \%$ bot-infected computers in the APJ region located in
China and $15 \%$ in Taiwan. Globally, $8 \%$ of infected computers are located in China and $3 \%$ in Taiwan.

Amongst the top five countries with the most bot-infected computers in the APJ region, China is the only country to have experienced a fall in the proportion of bot-infected computers. It is not clear if this reflects a real decrease in the number of bot-infected computers in China, or a corresponding increase in other countries in the APJ region.

Table 1 Bot-infected computer ranking in the APJ region in 2007

\begin{tabular}{|c|c|c|c|}
\hline Country & $\begin{array}{c}\text { Jul-Dec 07 } \\
\text { Rank, (\%) }\end{array}$ & $\begin{array}{c}\text { Jan-Jun 07 } \\
\text { Rank, (\%) }\end{array}$ & $\begin{array}{c}\text { Jul-Dec 07 } \\
\text { Global \% }\end{array}$ \\
\hline China & $1(43)$ & $1(78)$ & 8 \\
\hline Taiwan & $2(15)$ & $2(7)$ & 3 \\
\hline South Korea & $3(11)$ & $3(5)$ & 2 \\
\hline Japan & $4(6)$ & $4(2)$ & 1 \\
\hline Australia & $5(5)$ & $6(2)$ & 1 \\
\hline
\end{tabular}

Source: Symantec [1:13]

Symantec suggested that the decrease in the number of bot-infected computers in China might be "due to the significant reduction in the availability of many Web sites, such as Web forums and blogs, for several months in China during this period" $[1: 9,2]$. It might be attributed to the operation of the "Golden Shield Project" in the second half of 2007 and the meetings of the People's Representative Congress (人民代表大會) which was held in August to September of the year [2,3].

The fall in the number of bot-infected computers is seen as temporary and an aberration from the growth trend. According to Symantec [4], the average percentage of bot-infected computers in China rose from 43 per cent in the second half to an average of 58 per cent in 2008.

\section{METHODOLOGY}

The data used in this paper was collected in the Greater China Area (Taiwan, China and the Hong Kong Special Administrative Region). Thirty-eight interviews (including four focus groups, one in China and three in Taiwan) with a total of 44 interviewees were conducted in Taiwan and China during the years 2008 and 2009.

Interviewees were selected purposely based on their work experience or background. People with knowledge of information security and cybercrime were potential samples for this research. These included but were not limited to IT people in government agencies and private companies, police officers, prosecutors, and other professionals in cybercrime 
and information security, such as professors, managers of legal compliance in companies, and information security experts in big accounting firms which audit information security and conduct staff training in organisations. Interview data reached the point of data saturation.

In Taiwan, there were 23 interviews with 28 interviewees. There were only three interviewees with less than ten years experience in information security or related areas. All other interviewees in Taiwan had more than ten years working experience in this area. Four participants were female.

Among all the interviews in Taiwan, ten were conducted with 12 participants from the private sector; five interviews were conducted with seven participants from the public sector; four interviews were conducted with five participants from law enforcement agencies such as the police and prosecutors; and four professors were interviewed who were experts in law, information security issues or criminology.

In China, 15 interviews were conducted and 16 people participated. Seven interviews were conducted with eight participants from the private sector; three policemen were interviewed as well as five professors. With regard to the experience levels of the interviewees in China, only two had experience in the related area of less than ten years. In terms of gender, only one interviewee was female.

There were no government agencies willing to be interviewed. As well, there were no Chinese prosecutors interviewed. However, in lieu of formal prosecutors, some professors interviewed were also concurrently serving as deputy chief procurators. Their data helps overcome the lack of data from government officers and prosecutors in China.

All the interviewees interviewed in Taiwan were coded with the letter " $T$ " while those in China were coded with the letter " $C$ ". The number following the letter refers to the case record. For example, T001 means the first interview done in Taiwan.

\section{Why IS THERE A High PREVALENCE OF BOt-INFECTED COMPUTERS IN TAIWAN AND CHINA?}

Symantec found that Taiwan and China are the two countries with the most bot-infected computers in the APJ region. Possible reasons for this are considered below.

\section{A. Technical perspective: Number of Internet users and the speed of broadband}

There are a large number of Internet users in Taiwan and China. China is the country with the greatest number of Internet users around the world, while Taiwan has one of the highest densities of Internet users. Also, China and Taiwan are countries with a large number of Internet hosts.

Broadband access is also essential for bot program distribution. It needs fast broadband to disseminate phishing sites or spam. As the Internet in Taipei is now transmitted through fibre cable this is also considered to be facilitating factor [5].

\section{B. User perspective: Users' consciousness and ability to protect their computer}

Users' consciousness and knowledge of information security is also a vital element for computer security. If a user lacks an awareness of computer security, they may easily become a victim. When using their computers, they are more likely to open an email or a file containing malware, or to click on a link sent by friends whose computers are already bot-infected.

CNCERT/CC [6] mentioned that Chinese users' lack of safety awareness and their lack of knowledge about how to protect their computers are two reasons for botnet problems in China. C011 takes the similar view that users should be responsive to the botnet issue:

The reason why bot-infected computers are common in China can be explained by two reasons: first, most users do not have enough knowledge on information security; second, most users do not update or fix the patch regularly (C011_01).

This is not only the case for general users. C009 said that even users in government agencies do not have much cybercrime sense. In addition, when faced with cybercrime problems, government officials are not enthusiastic about dealing with them.

Taiwanese information security experts believed that the same reasons explained the flood of botnets into Taiwan. T002, T005, T017, T019 and T022 all said client ignorance of information security is the main reason for the flood of botnets into Taiwan. T022 said: "According to my experience, lots of people have insufficient information security protection".

T017 expressed the same problem and further explained the "social engineering" modus operandi used to trap users:

Actually, when our systems are infected or embedded with Trojans or bots, it can usually be blamed on the bad habits of our employees. They might be curious and attracted by a fancy letter title and will open emails sent by their friends or someone who pretends to be their friend. The fancy letter title might be something related to work or a current and critical issue, or even some porn news or sexy photos of an actress, like Lin Zhi-lin (林 志玲), the beautiful model in Taiwan. However, it is a kind of social engineering method (T017_02).

T002 and T008 mentioned that the method of social engineering differs depending on the information that attackers require. If an attacker is targeting a specific government agency, he might use an email with a title related to that government agency. Sometimes, the attacker might possess information relating to the victim which he could use to win the trust of the victim and encourage him to open the email (T002and T008).

When users discover that their computer is infected, they usually reinstall the operating system. This prevents the computer from being controlled by a botnet controller and usually makes their computer run more efficiently and fast. However, this does not put a stop to their future 'zombie-career.' In Shi [5], the senior information security consultant said that reinstalling the operating system will only offer protection from botnet manipulation for a short period of time. Without a knowledge and sense of computer security, Trojans or bot programs will soon again infect the computer. 
T002 expressed his concern that users are unwilling to pay for hardware or software related to information security protection. This might be another reason why users are victimised repeatedly:

Indeed, although they know the impact of cybercrime and information security problems, only a few of them are willing to invest in protecting their hardware and software relating to information security (T002_16).

However, it is important to realise that the notion of hacking is different to the break-in in the real world. When a break-in happens in the real world, that might only cause loss to the owner, however, a hack-in into a computer might cause problems beyond the owner of the computer. It might also have effects on the public.

As T007 said, hackers are not only interested in stealing data from computers they hack into. The hacker will do more than that and may use the computer as a springboard to hack into other systems. T002 somewhat extended T007's words and said that individual computers could be used in serious cyber attacks. He used as an example the 2007 DDoS experience of Estonia which paralysed critical infrastructure:

It is easy to hack into an individual's computer. And those who hack into an individual's computer might not be interested in cyber-terrorism. However, when there is someone who has bad intentions and is able to control these computers, these computers will become his soldiers. There might be hundreds of thousands of soldiers under his command and control that can launch a DDoS attack and block certain critical infrastructure, just as was done to the public systems in Estonia (T002_10).

In light of this, T002 said that education on information security is lacking and every hacking event should be taken seriously, no matter whether it targeted individuals, companies or government agencies.

\section{Political perspective: The special political situation between Taiwan and China}

The situation above might be seen as common throughout the entire computer world. However, there are some unique features that contribute to the bot problems in Taiwan and China. Symantec argues that the political situation between Taiwan and China may contribute to the extent of malicious activity between these countries $[1,7]$.

T002 and T021 both said that political tension between Taiwan and China was the key cause of serious botnet problems in both locations (T002, T011-2, T021, C003, and C014). T020 said most cyber attacks on Taiwan originated from a China cyber army1, or Wang Jun（網軍）in Mandarin (T020). Some interviewees indicated said the main reason why China installed Trojan and bot programs onto Taiwanese or Chinese computers, especially government systems, was to steal classified or sensitive data and avoid investigation (T018, T017, T015, T004, T013, and C012).

The acting director of the National Security Bureau in Taiwan has said that a Chinese cyber army launched more than 3,100

1 Here, a cyber army, called Wang Jun in Mandarin, generally means the hacking that is initiated with the government's direct or indirect support. attacks against Taiwanese Government systems in 2008. This does not include attacks against the private sector. Their purposes were mainly related to stealing data and sensitive information [8].

However, some hackers do it just for fun or revenge. They simply try to take over the command and control power of the user (T003_02, T004_05, T013_02, T014_04, T019_03, and C003_08). ${ }^{-}$T019 mentioned that a website he managed was once hacked and a Chinese national flag (usually called the "five star flag' in Taiwan) was inserted on the main page and malware was installed:

Our website was once hacked. This happened before I took this position. They put the 'five star flag' on the main page. I reckon this was done by Chinese hackers. Our organisation scanned the whole system afterwards, and it is said they found some malware in the system (T019_03).

\section{Cultural perspective: Sharing culture and language}

As mentioned earlier, "social engineering" is an important skill used by hackers. If they have appropriate "social engineering" skills, they can easily disseminate bots or Trojans by encouraging a user to open an email or an attached file. Nonetheless, "social engineering" relies heavily on the use of language and a background understanding of culture.

For instance, T017 said if a hacker knows what is happening in a culture, or is aware of the critical issues affecting a society, or if they can respond to current trends (for example, who are the hottest celebrities in Taiwan and China), it will be easier for the hacker to succeed in spreading bots or Trojans.

Although an understanding of culture is desirable, language is another important element. Consistent with Symantec's evidence of Chinese-language based malware [1, 4], T011-1 indicated the reason why Internet users in Singapore are not suffering as much as Taiwanese and Chinese users from Chinese-language based malware:

...Although Singapore is a bi-lingual society of Chinese and English, everyone is using English; official documents are written and published in English. But we (Taiwanese) are using the same language as China (T011-1_18).

Indeed, if users in Taiwan or China received an email written in English, they are unlikely to read it because most of the population does not have good English language skills. This easily explains why messages purporting to be from African or Middle-eastern princes needing help are less successful in Taiwan and China.

\section{E. Business perspective: Attitude of information security enterprises}

The attitude of information security enterprises is an important factor highly correlated to botnet problems between Taiwan and China. Some bots and Trojans are designed only for attacking Chinese systems $[1,4]$. Symantec and Trend Micro found a specific modelling malware which attacks Taiwanese websites only:

Symantec named the malware W32.Bacalid, which is a typical "modelling malware". Specifically, W32.Bacalid is able to recognise simplified Chinese characters and bypass them. [9]. 
T002 and C005 addressed the same issue. C005 said a proper tool to control the botnet problem is lacking in Taiwan and China (C005 17). T002 also said that another reason why Taiwan and $\bar{C}$ hina have so many bot-infected computers is that that some malicious activity focuses only on the Chinese system, especially those used by the Taiwanese and Chinese Governments (T002_17).

However, T002, C004, C009, and C014 pointed out that large information security companies only invest limited resources to research threats to Taiwan and China. T002 said that a large number of users do not purchase a licensed version of their software:

Based on the goal of increasing their profit and revenue, information security companies are not willing to customise or invest in developing anti-virus or anti-hacking software that is used to guard against malicious activity targeting Chinese or Taiwanese systems (T002_18).

He further explained that because researchers are rated by how much malware they can detect, they are not always willing to analyse threats which only target a specific country or system:

Those big information security companies evaluate their researchers by how much malware they can detect and the number of cases reported. As a consequence, researchers focus their analyses on the main threats occurring in western countries, even if these researchers are based in Taiwan or China (T002_18).

\section{CONCLUSION}

This paper examined the reasons that caused China and Taiwan to become the two countries with the most bot-infected computers in the APJ region. From the technical perspective, both Taiwan and China have large numbers of Internet users and Internet hosts. Also, the high-speed internet used in the area helps in transmitting bot programs.

The special political situation between Taiwan and China also attributes to the prevalence of bot-infected computers in Taiwan and China. There is some evidence of political motivation behind attacks on government computers. Further, with similar culture and language, social engineering methods can successfully attract people in Taiwan and/or China to download bot-programs.

Finally, the specific nature of some malicious activity occurring in Taiwan and China means that it may not attract the attention of researchers in large information security companies, which can result in a lower level of development of technical counter-measures. Further, most users lack the consciousness and ability to protect their computers and there are therefore not enough protective measures in place to protect against malware which is targeting Chinese computing systems. Users often do not know how to protect their systems, or if they know, they might not have sufficient access to computer tools to protect their system. The result is an increased exposure to the threat of botnet activity, which affects security both at the individual and national level.

\section{REFERENCES}

[1] Symantec, Symantec APJ Internet Security Threat Report XIII: Trend for July - December 2007. Cupertino, CA: Symantec Corporation, 2008.

[2] A. Olesen, "China tightens Internet controls: Content under scrutiny in run-up to sensitive Communist Party Congress." Internet: http://www.msnbc.msn.com/id/21268635, Oct. 14, 2007 [Mar. 23 2009].

[3] J.Y. Su, "Golden Shield Project-Everyone in China is under the surveillance." Internet: http://www.libertytimes.com.tw/ 2008/new/dec/9/today-fo5.htm, Dec. 9, 2008 [Mar 15, 2009].

[4] Symantec, Symantec APJ Internet Security Threat Report XIII: Trend for 2008 Cupertino, CA: Symantec Corporation, 2009.

[5] W.B. Shi, "Taipei becomes the headquarter of bots in the Asia and Pacific Region.” Internet: http://www.ithome.com.tw/itadm/ article.php?c=54571, Apr. 20, 2009 [Aug. 20, 2009].

[6] CNCERT/CC, CNCERT/CC Annual report 2007. Beijing: CECERT/CC, 2008.

[7] Symantec, Symantec APJ Internet Security Threat Report XII: Trend for January- June 2007. Cupertino, CA: Symantec Corporation, 2007.

[8] S.C. Xu, "Over 3,100 cyber attacks towards Taiwanese Government System were originated by Chinese cyber army." Internet: http://www.libertytimes.com.tw/2009/new/mar/24/today-fo2.htm, Mar. 24,2009 [Sept. 21, 2010].

[9] Y.S. Yau, "Specific modelling model found which is targeting Taiwan only." Internet: http://www.ithome.com.tw/itadm/ article.php?c=39235, Sept. 5, 2006 [Aug. 27, 2009]. 